\title{
Intracoronary Glycoprotein IIb/IIIa Inhibitors Improve Short-Term Mortality and Reinfarction in East Asian Patients with ST-Segment Elevation Myocardial Infarction after Thrombus Aspiration: A Meta-Analysis
}

\author{
Jia-hong Wu, ${ }^{1}$ Pan-pan Hao, ${ }^{2}$ Yu-guo Chen, ${ }^{3}$ and Rui-jian Li $\mathbb{D D}^{3}$ \\ ${ }^{1}$ Department of Radiology, Qilu Hospital of Shandong University, Jinan 250012, China \\ ${ }^{2}$ Department of Cardiology, Qilu Hospital of Shandong University, Jinan 250012, China \\ ${ }^{3}$ Department of Emergency, Qilu Hospital of Shandong University, Jinan 250012, China \\ Correspondence should be addressed to Rui-jian Li; liruijian2003@sdu.edu.cn
}

Received 13 April 2018; Revised 15 July 2018; Accepted 30 July 2018; Published 15 August 2018

Academic Editor: Vincenzo De Feo

Copyright (c) 2018 Jia-hong Wu et al. This is an open access article distributed under the Creative Commons Attribution License, which permits unrestricted use, distribution, and reproduction in any medium, provided the original work is properly cited.

\begin{abstract}
Objective. Intracoronary (IC) glycoprotein IIb/IIIa inhibitors (GPIs) after thrombus aspiration (TA) for patients with ST-segment elevation myocardial infarction (STEMI), as compared with percutaneous coronary interventions (PCI) alone, is still on debate. To address this issue, we performed a meta-analysis of results from prospective or randomized controlled trials on the topic. Methods. We searched electronic and printed sources (up to June 20,2016) according to the selection criteria. Data were abstraction and meta-analysis was performed using RevMan 5.3 software. Results. The cohorts involved 14 articles describing 1,918 participants were included. The incidence of the short-term major adverse cardiac events (MACE) was significantly reduced with intracoronary GPIs after TA (odds ratio [OR]: $0.29 ; 95 \%$ confidence interval [CI]: 0.13 to $0.65, \mathrm{p}=0.003$ ). Benefits were noted for short-term mortality (OR: $0.31 ; 95 \%$ CI: 0.17 to $0.57, \mathrm{p}=0.0002$ ) and reinfarction (OR: $0.28 ; 95 \%$ CI: 0.10 to $0.78, \mathrm{p}=0.01$ ) in subjects who received intracoronary GPIs after TA. Moreover, the Thrombolysis in Myocardial Infarction (TIMI) trial grade 3 postprocedure (OR: 2.29; 95\% CI: 1.72 to 3.04, $\mathrm{P}<0.00001)$ and complete ST-segment resolution (STR) rate (OR: 2.68; 95\% CI: 1.85 to 3.87, P<0.00001) were both improved with intracoronary GPIs after TA. As a result, left ventricular ejection fraction (LVEF) at short-term follow-up showed a significant difference (OR: 7.33; 95\% CI: 5.60 to 9.06, p<0.0001) in favor of the TA and intracoronary GPIs administration. Conclusions. Our study demonstrates that intracoronary GPIs may have a synergistic effect with thrombus aspiration on short-term mortality, reinfarction, and cardiac functional recovery.
\end{abstract}

\section{Introduction}

Primary percutaneous coronary intervention (PCI) has become the preferred reperfusion modality for patients with acute ST-segment elevation myocardial infarction (STEMI) [1]. As we all know, the possibility of distal embolization of atherosclerotic plaque and thrombus with subsequent microvascular injury and increased infarct size during primary PCI is associated with adverse cardiovascular events [2]. Thrombus aspiration (TA) has the potential of reducing distal embolization and improving microvascular perfusion during primary PCI. Even though numerous international studies have been reported, there are still conflicting results on the clinical impact of thrombus aspiration during primary PCI [3, 4]. Recent evidence from Routine Aspiration Thrombectomy With Percutaneous Coronary Intervention (PCI) Versus PCI Alone in Patients With ST-Segment Elevation Myocardial Infarction (STEMI) Undergoing Primary PCI (TOTAL) trial, the largest trial of thrombus aspiration in STEMI so far, suggested that routine thrombus aspiration, as compared with PCI alone, did not reduce the risk of major adverse cardiovascular events (MACE) within 180 days [5], consistent with those of Thrombus Aspiration during STSegment Elevation Myocardial Infarction (TASTE) trial [4] and the Intracoronary Abciximab and Aspiration Thrombectomy in Patients With Large Anterior Myocardial Infarction 
(INFUSE-AMI) trial [6]. However, TA along with intracoronary (IC) glycoprotein IIb/IIIa inhibitors (GPIs) was associated with improved 30-day mortality in INFUSE-AMI trial [6], which suggested the synergistic effect of TA and GPIs might be attributed to improvement in clinical outcomes. On the other hand, some East Asian studies (especially in China) from the year 2008 to 2015 yielded conflicting or inconclusive results $[6,7]$. The reason for the discrepancy is unclear but may be related to low statistical power or difference among the ethnic groups studied.

In this meta-analysis, we aim to assess the effects of intracoronary GPIs after thrombus aspiration compared with PCI alone in STEMI patients from the year 2008 to 2015.

\section{Methods}

2.1. Data Sources and Searches. We performed a systematic search for articles in the databases MEDLINE (via PubMed), EMBASE, and the Cochrane Library (Cochrane Central Register of Controlled Trials) up to June 20, 2016, using the following keywords: (thrombus aspiration) AND (intracoronary) AND \{("abciximab"[Substance] or "abciximab”[All Fields]) or ("eptifibatide"[Substance] or "eptifibatide"[All Fields]) or ("tirofiban"[Substance] or "tirofiban"[All Fields]\}. We also searched the China National Knowledge Internet database to retrieve relevant studies published in Chinese. We restricted the search to human studies but not language. Further articles were retrieved by a manual search of references from recent reviews and relevant published original studies. Studies were screened by reading the abstracts and titles and then selected after reading the full text.

2.2. Study Selection. A study was selected if (1) the subjects were prospectively or randomly assigned to TA plus GPIs or PCI alone in a parallel-group design; (2) major adverse cardiac events were reported as outcomes; (3) GPIs were administrated by intracoronary during the procedure. We excluded studies that were cross-sectional or case-control designs. In case of duplicate publication, we chose the publication reporting on the primary analysis. The long-term clinical outcome was defined as more than three months, and the short-term clinical outcome was less than three months or in hospital.

2.3. Data Extraction and Quality Assessment. Data were extracted independently by 2 investigators (Li R.J. and Hao P.P.) using a standardized extraction form and compared. Discrepancies were resolved by discussion with a third investigator (Chen Y.G.) and by referencing the original report. The grade of study quality was assessed as the previous metaanalysis $[8,9]$.

2.4. Data Analysis. RevMan 5.3, developed by the Cochrane Collaboration (http://tech.cochrane.org/revman, released on 13 June 2014), was used for the meta-analysis. Heterogeneity was tested with the chi-square and $I^{2}$ tests. Statistical significance was a 2 -tailed $P<0.05$. Results showing no significant differences were analyzed by the fixed effects model and those showing significant differences were analyzed by the
DerSimonian-Laird random effect model. We also performed a sensitivity analysis to explore the robustness of our results. For MACE, mortality, and reinfarction, we evaluated publication bias using funnel plots and the fail-safe number with $P<0.05$ (Nfs0.05), Nfs0.05 $=(\Sigma \mathrm{Z} / 1.64)^{2}-\mathrm{k}$, where $\mathrm{k}$ is the number of studies included in the meta-analysis. Any calculated Nfs value smaller than the number of observed studies indicated publication bias that might influence the meta-analysis results.

\section{Results}

A total of 14 observational studies with 1,918 participants were finally included (Figure 1) [7, 10-22]. The geographical distribution of 14 studies was all in East Asian regions. One study was from the Republic of Korea, 1 from Taiwan region, and the others from the mainland of China. Of 14 articles, 11 studies [10-20] reported the short-term clinical outcome; other 3 studies $[7,21,22]$ reported the long-term clinical outcome (18 months, 12 months, and 6 months, respectively). Figure 1 reported study selection procedure, while Table 1 summarizes the most relevant characteristics of the selected studies.

Seven studies depicting baseline characteristics were summarized in Table 2. For these studies, baseline characteristics were not significantly different between the two groups. Choi's study was an abstract from ANGIOPLASTY SUMMIT in 2009 [22], and we could not obtain accurate baseline characteristics although we have contacted the corresponding author. Other 6 studies described those baseline characteristics were balanced between two groups in papers, while no accurate data of baseline characteristics were obtained from [15-20].

There were 8 studies which reported short-term MACE after the procedure $[10-12,14,16-18,20]$. The analysis for the short-term MACE revealed that the incidence of MACE was significantly lower in the patients treated with intracoronary GPIs after TA than those with PCI alone (1.86\% versus $6.06 \%$; odds ratio (OR): $0.29 ; 95 \%$ confidence interval (CI): 0.13 to $0.65, \mathrm{p}=0.003$; Figure $2(\mathrm{a}))$. In terms of short-term mortality, 7 studies reported the results [11$13,15,16,18,19]$. The incidence of short-term mortality was significantly reduced in subjects who received TA and IC GPIs treatment (3.06\% versus $8.59 \%$; OR: 0.31 ; $95 \%$ CI: 0.17 to $0.57, \mathrm{p}=0.0002$; Figure $2(\mathrm{~b}))$. Eight studies reported the short-term reinfarction rates $[10,12-16,18,19]$. An obviously decreased risk of short-term reinfarction was observed in the TA and IC GPIs group compared with the PCI group (0.85\% versus $3.37 \%$; OR: 0.28 ; $95 \% \mathrm{CI}$ : 0.10 to $0.78, \mathrm{p}=0.01$; Figure 2(c)).

Looking at the long-term MACE reported by 3 studies, our analysis did not show a significant difference between the two groups (6.41\% versus $9.65 \%$; OR: $0.69 ; 95 \%$ CI: 0.20 to $2.34, \mathrm{p}=0.55$; Figure $3(\mathrm{a}))[7,21,22]$. Similarly, no significant difference was noted in long-term mortality between the two groups (1.28\% versus 6.81\%; OR: 0.94 ; $95 \% \mathrm{CI}$ : 0.11 to 8.04 ; $\mathrm{p}=0.95$; Figure $3(\mathrm{~b}))[7,21,22]$. The analysis of long-term reinfarction rate was unable to be performed due to only two studies reporting $[7,21]$. 


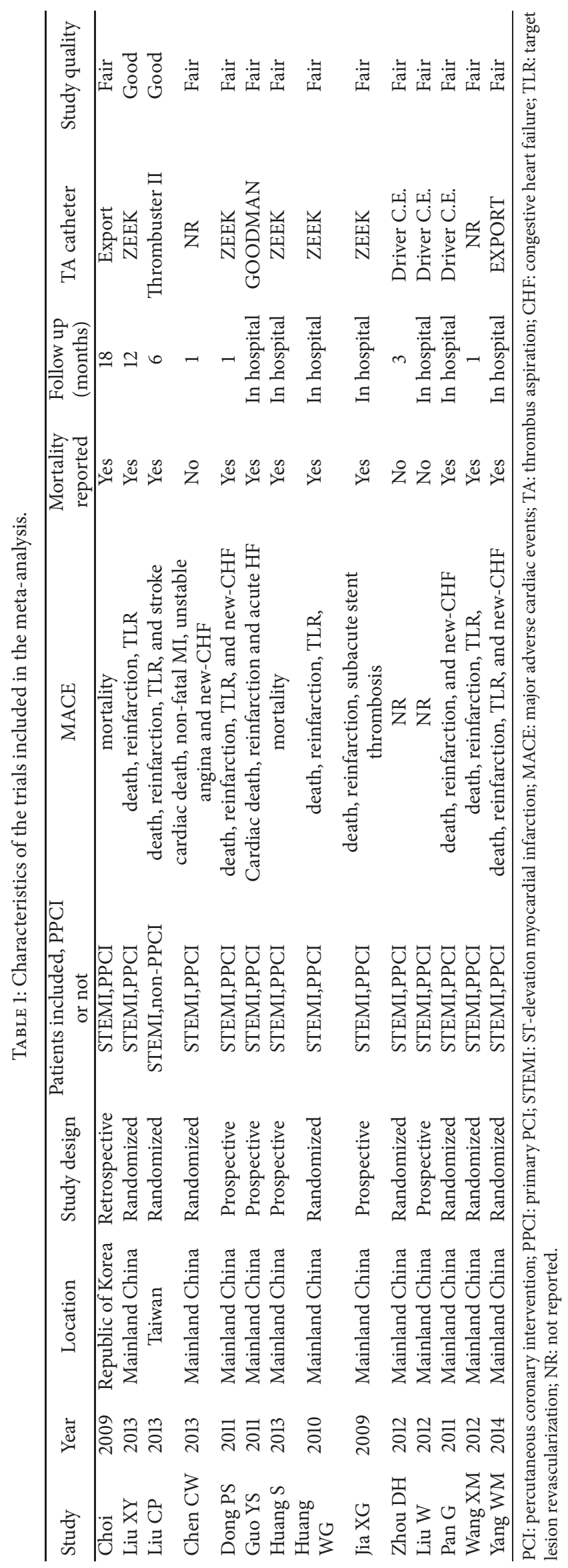




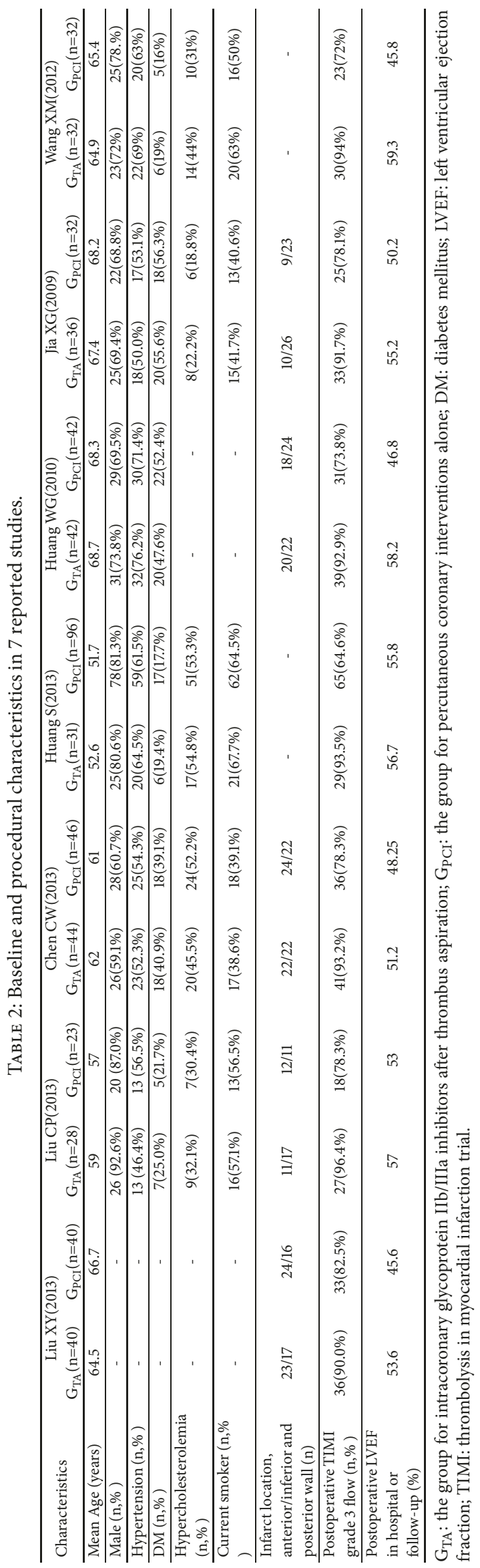




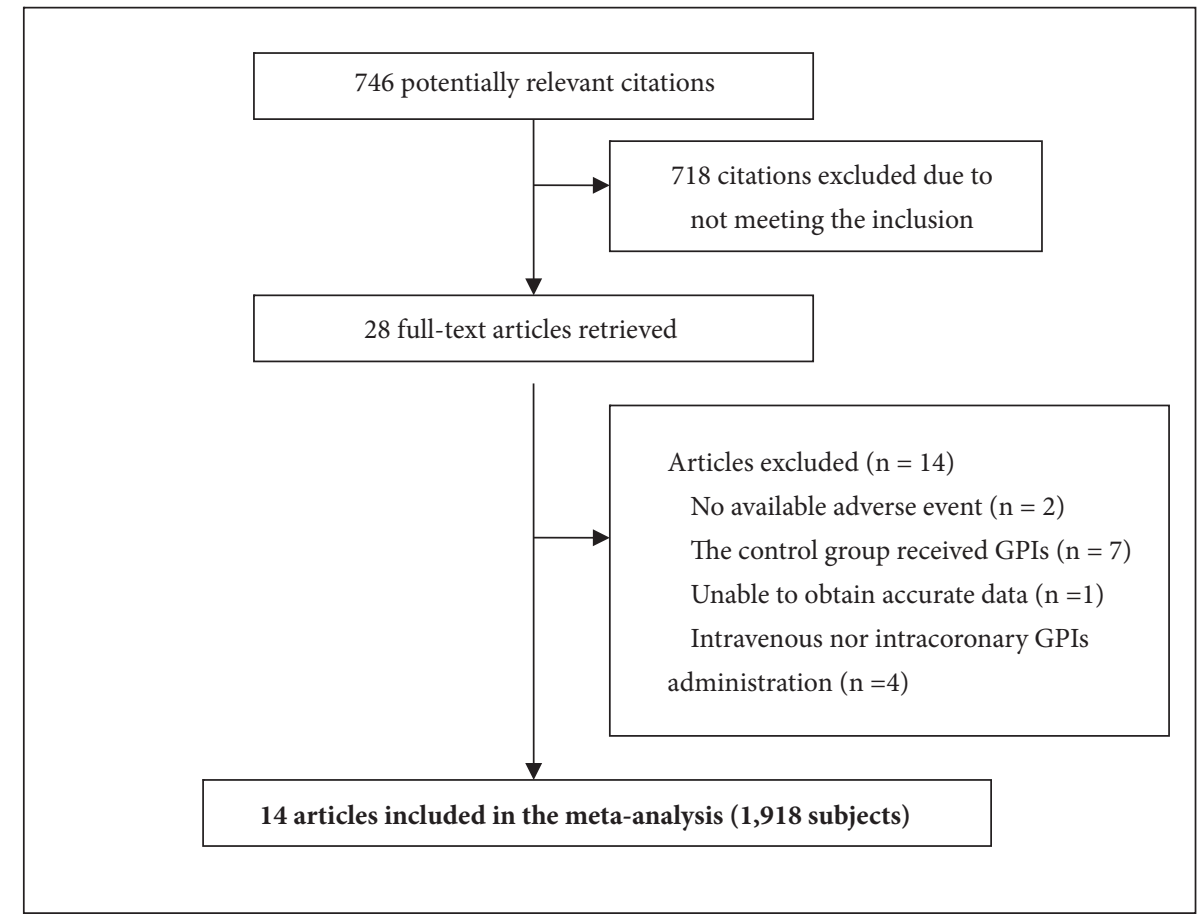

FIGURE 1: Selection of articles for the meta-analysis. GPIs: glycoprotein IIb/IIIa inhibitors.

TABLE 3: Subgroup meta-analysis of postoperative TIMI grade 3 flow and MACE according to TA catheter.

\begin{tabular}{lcccc}
\hline \multirow{2}{*}{ TA catheter } & \multirow{2}{*}{ Number of studies } & \multicolumn{2}{c}{ Postoperative TIMI grade 3 flow } & \multicolumn{2}{c}{ MACE } & OR $(95 \%$ CI $)$ & P value & OR $(95 \%$ CI $)$ & P $=0.05$ & $0.22(0.14,0.35)$ \\
\hline ZEEK & 5 & $2.52(1.01,6.31)$ & $\mathrm{P}=0.0004$ & $0.22(0.02,2.14)$ \\
\hline Driver C.E. & 3 & $4.97(2.03,12.15)$ & $\mathrm{P}=0.0001$ & $0.38(0.14,1.0)$ \\
\hline EXPORT & 2 & $8.75(2.92,26.26)$ & $\mathrm{P}=0.19$ \\
\hline
\end{tabular}

TA: thrombus aspiration; OR: odds ratio; 95\% CI: 95\% confidence interval; TIMI: thrombolysis in myocardial infarction trial; MACE: major adverse cardiac events.

Thirteen studies reported the postprocedural flow grades based on the Thrombolysis in Myocardial Infarction (TIMI) trial $[7,10-15,17-22]$. The incidence of postprocedural TIMI flow grades 3 was higher in patients treated with TA and IC GPIs compared with those who did not (81.9\% versus $63.6 \%$; OR: 2.29 ; $95 \%$ CI: 1.72 to $3.04, \mathrm{P}<0.00001$; Figure $4(\mathrm{a}))$. Seven studies reported complete ST-segment resolution (STR) rate at 60 minutes 90 minutes after the procedure $[7,11,12,14,16$, $20,22]$. The incidence of postprocedural complete STR significantly increased in patients treated with TA and IC GPIs (79.8\% versus 59.2\%;OR: 2.68; 95\% CI: 1.85 to 3.87, $\mathrm{P}<0.00001$; Figure 4(b)). Subgroup analysis, according to TA catheter, showed that both postprocedural TIMI flow 3 and MACE were improved in studies using ZEEK aspiration catheter (Zeon Medical Inc., Tokyo, Japan) and EXPORT aspiration catheter (Medtronic, Minneapolis, Minnesota), whereas no benefit of MACE was observed in studies using Driver C.E. aspiration catheter (Invatec, Brescia, Italy) (Table 3).

Importantly, the analysis of left ventricular ejection fraction (LVEF) before discharge or at short-term follow-up (reported by 11 studies) showed a significant difference $(54.5 \%$ versus $47.0 \%$; OR: 7.33; 95\% CI: 5.60 to $9.06, \mathrm{p}<0.0001)$ in favor of the TA and IC GPIs administration route (Figure 4(c)) [7, 10-14, 16, 18-21]. There were no significant differences in the major bleeding and minor bleeding events between the two groups (4.22\% versus 3.77\%; OR: $1.16 ; 95 \%$ CI: 0.63 to $2.15, \mathrm{p}=0.64$; Figure 5) $[7,12-19,21]$.

In heterogeneity testing and sensitivity analysis, we also found no significant heterogeneity for studies reporting short-term MACE, death, and reinfarction, and exclusion of any single study did not alter the overall finding. The funnel plot assessing the publication bias is shown in Figure 6. We calculated the Nfs0.05 for MACE, death, and reinfarction. The comparative Nfs0.05 for MACE, death, and recurrent MI of the short term was $10.29,14.93$, and 2.97 , whereas those of long term were $-0.99,-0.99$, and -0.82 , which indicated publication bias that might influence the meta-analysis results.

\section{Discussion}

The main findings of the present meta-analysis are as follows: (1) a combination of thrombus aspiration and intracoronary GPIs seemed to be superior to PCI alone in terms 


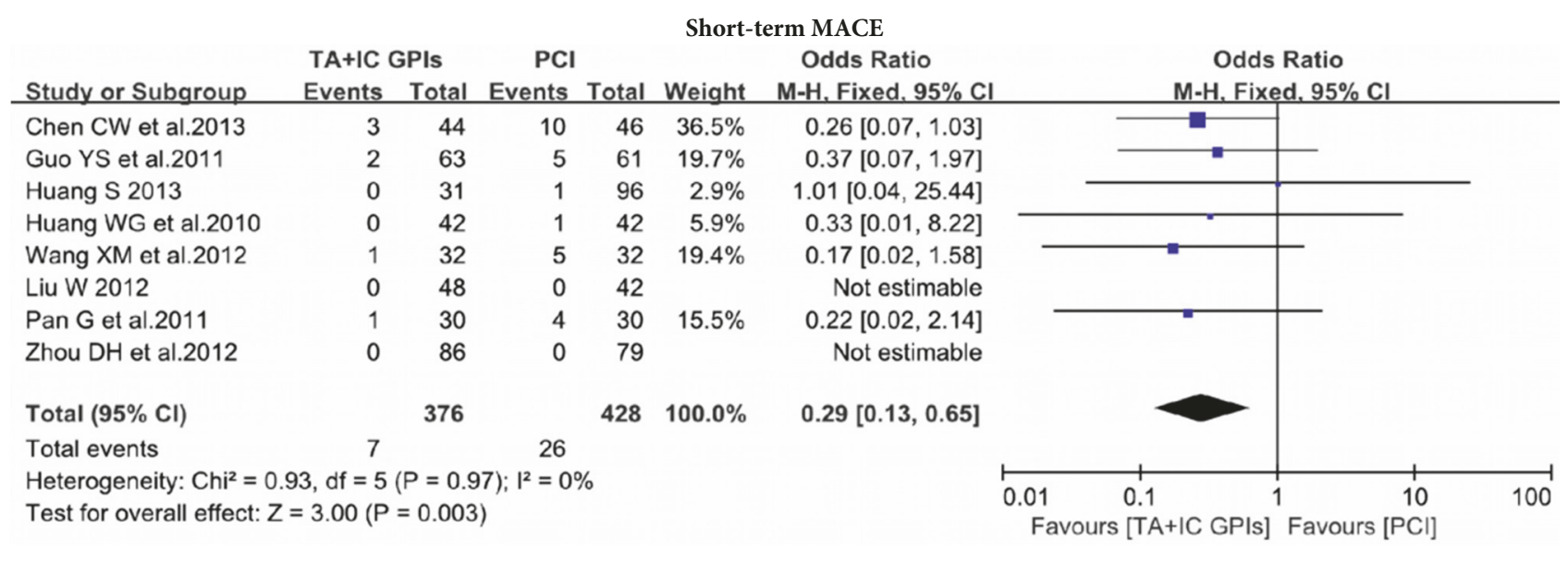

(a)

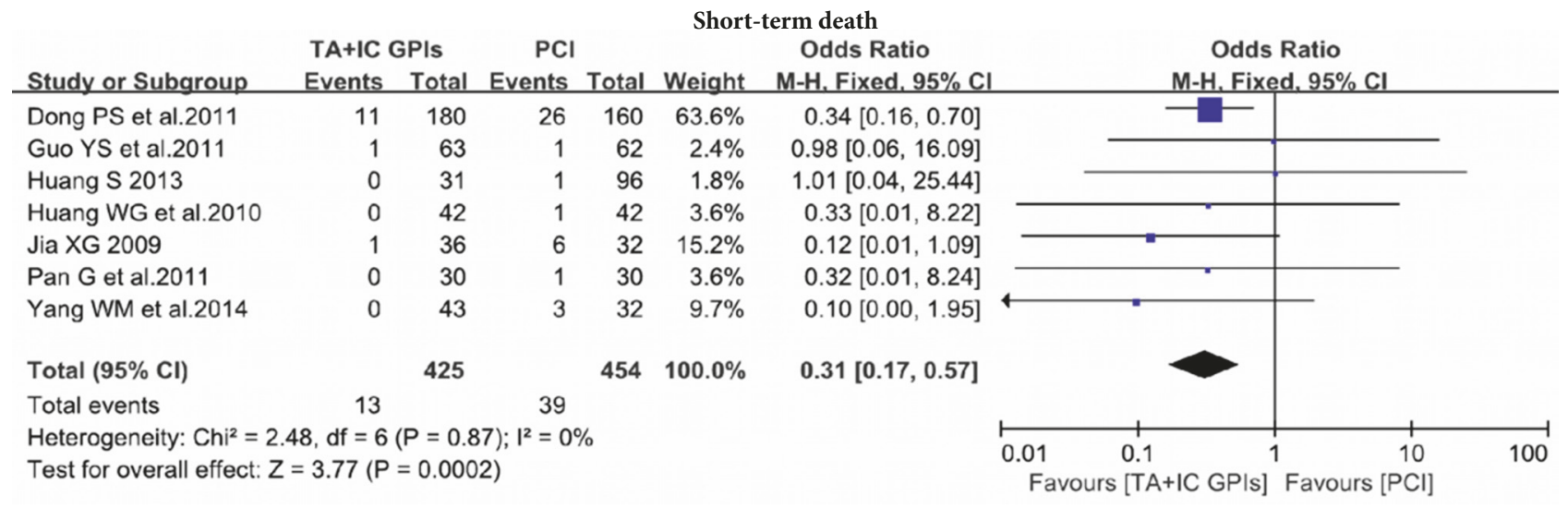

(b)

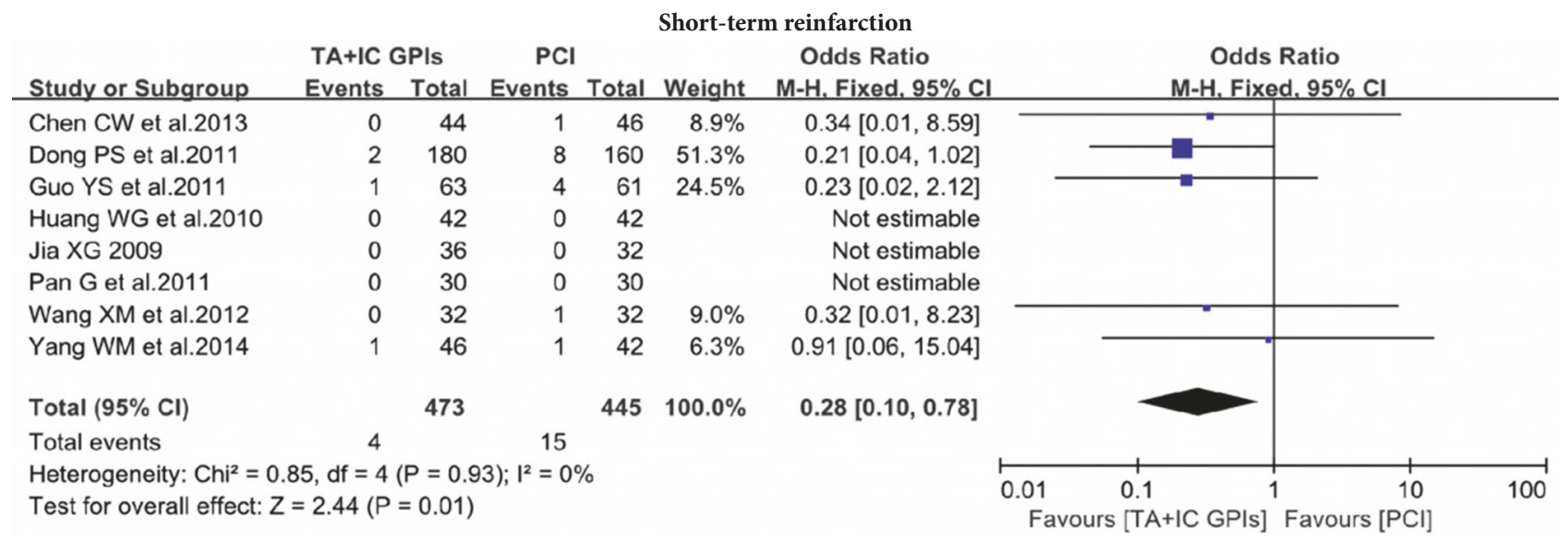

(c)

Figure 2: (a) The meta-analysis of MACE at the short-term follow-up; (b) the meta-analysis of death at the short-term follow-up; (c) the meta-analysis of reinfarction at the short-term follow-up. MACE: major adverse cardiac events; TA: thrombus aspiration; IC: intracoronary; GPIs: glycoprotein IIb/IIIa inhibitors; PCI: percutaneous coronary interventions.

of enhancing myocardial perfusion, as assessed by postprocedural TIMI flow 3, and complete STR rate. Importantly, cardiac function at short-term follow-up, analyzed by LVEF, showed much better to be in the thrombus aspiration and intracoronary GPIs group over the PCI group.
(2) The incidence of short-term MACE was significantly reduced with intracoronary GPIs after thrombus aspiration, including death and reinfarction, whereas there was no trend towards better outcome in studies with long-term MACE. 


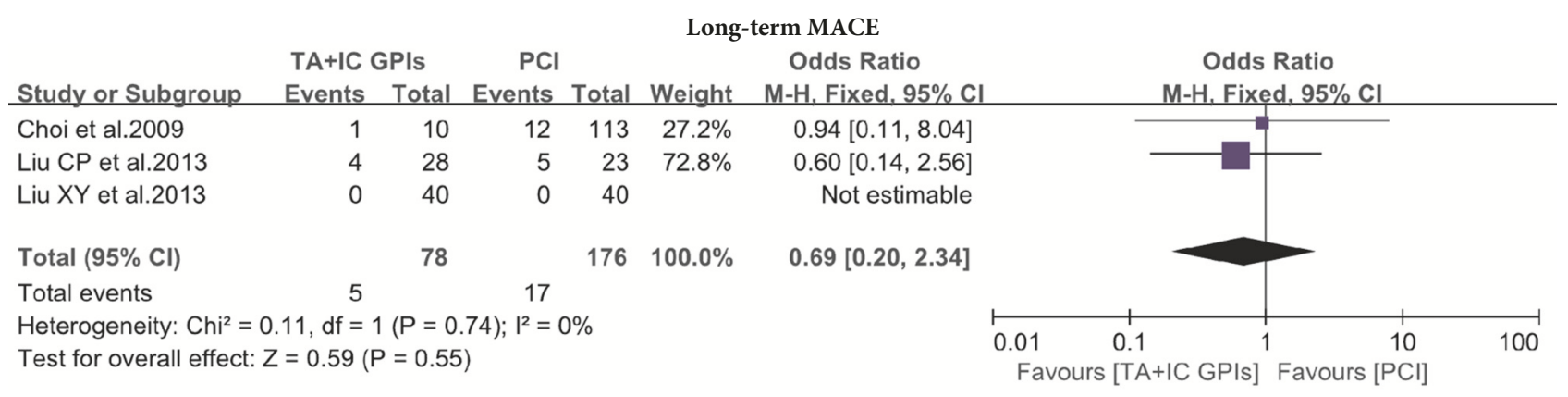

(a)

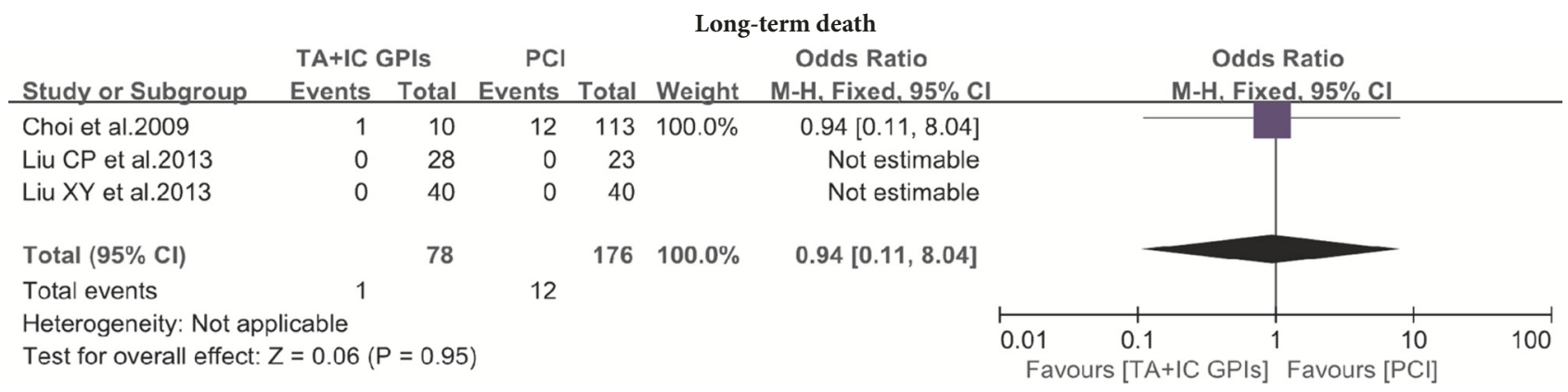

(b)

FIgURE 3: (a) The meta-analysis of MACE at the long-term follow-up; (b) the meta-analysis of death at the long-term follow-up. MACE: major adverse cardiac events; TA: thrombus aspiration; IC: intracoronary; GPIs: glycoprotein IIb/IIIa inhibitors; PCI: percutaneous coronary interventions.

Thrombus aspiration during primary PCI is controversial, especially after the TOTAL trial which showed that routine thrombus aspiration did not reduce the risk of longterm MACE, as compared with PCI alone, and the findings are consistent with those of the INFUSE-AMI trial. However, a subgroup analysis of the INFUSE-AMI trial showed that thrombus aspiration plus intracoronary administration of GPIs improved myocardium perfusion and resulted in a better clinical prognosis [6]. A recent meta-analysis, summarizing the conflicting randomized controlled trials (RCTs) of comparing thrombus aspiration with the control arm, showed that thrombus aspiration along with GPIs is associated with improved 30-day mortality [23]. However, the authors performed metaregression and compared the studies with a higher proportion of glycoprotein IIb/IIIa inhibitor use and those with lower glycoprotein IIb/IIIa inhibitor use in the thrombus aspiration arm [23]. In contrast, we pooled the studies which directly compared thrombus aspiration plus GPIs with PCI alone, especially intracoronary administration. Our meta-analysis could demonstrate a reduced incidence of short-term MACE in the intracoronary GPIs after thrombus aspiration group, as compared to the PCI group. Further analysis showed that the benefit comes from reduced short-term mortality and reinfarction. Nonetheless, it has to be underlined that no benefit of long-term MACE was observed.

Ahn et al. found that distal embolization was less likely to occur in patients undergoing intracoronary abciximab and thrombus aspiration as assessed by on-site measurement of the index of microcirculatory resistance (IMR) after primary PCI [24], which suggested that combination treatment using GPIs and thrombus aspiration may synergistically improve myocardial perfusion in patients with STEMI undergoing primary PCI. It was suggested that direct intracoronary injection of GPIs might be superior to its intravenous injection for improving myocardial perfusion due to creating a higher local concentration of around the coronary thrombus, and high local concentration may facilitate thrombus disaggregation and improve microvascular perfusion [24, 25]. Our meta-analysis found that intracoronary GPIs after thrombus aspiration provided significant benefits in postprocedural TIMI flow grade 3, complete STR when compared with PCI alone. STR is a simple and reliable marker of effective myocardial reperfusion which correlates with cardiac functional recovery [26]. In the present meta-analysis, complete STR tends to be better in the intracoronary GPIs after thrombus aspiration group than that in the PCI alone group. This result coincided with the improvement in left ventricular function measured by LVEF and resulted in a trend towards better clinical outcomes.

A subgroup analysis of INFUSE-AMI [6] and a metaanalysis [27] suggest that a combination of thrombus aspiration and GPIs treatment is effective in decreasing infarct size and mortality as compared to each treatment alone or PCI alone. These findings are consistent with those of our meta-analysis. If most thrombotic materials are retrieved by thrombus aspiration catheter, GPIs could further dissolve residual thrombus and microemboli in the microvasculature. 


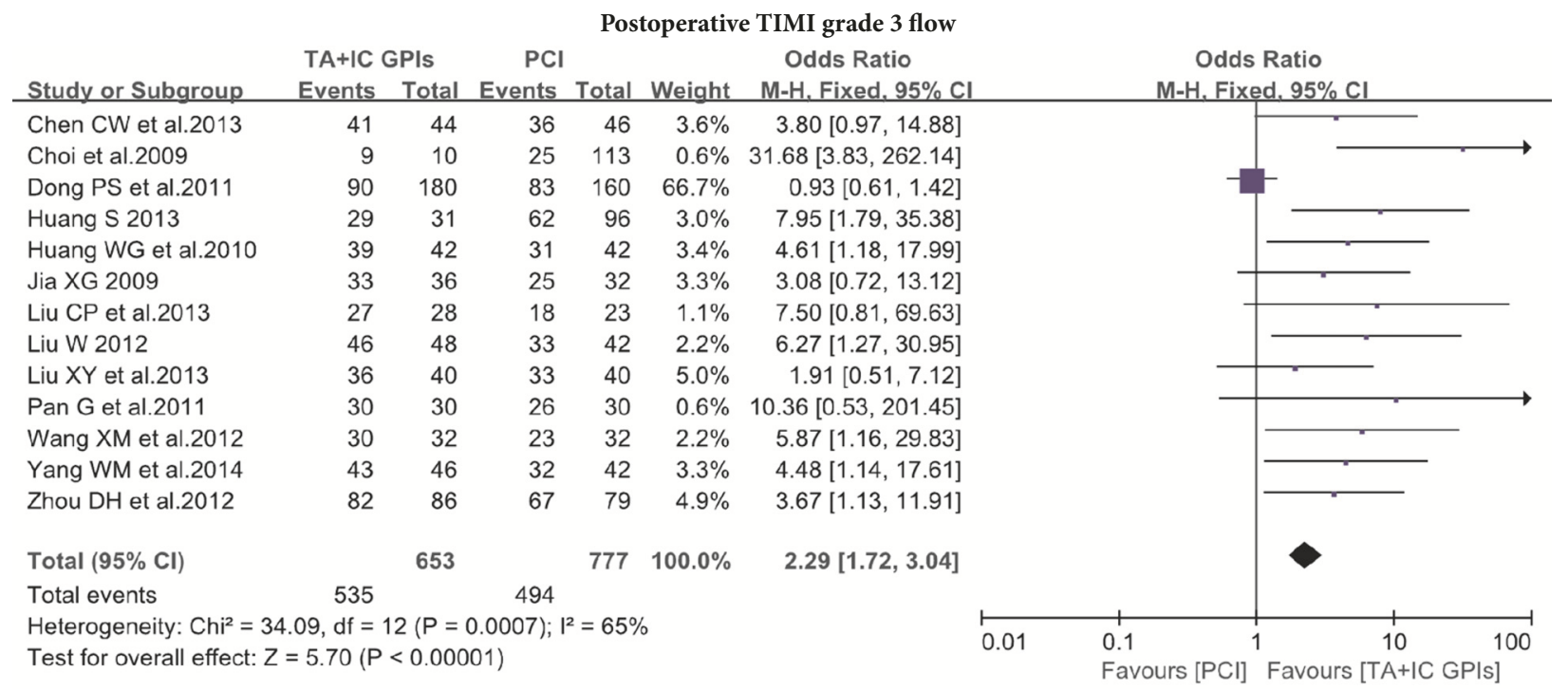

(a)

Complete ST resolution

\begin{tabular}{|c|c|c|c|c|c|c|c|c|c|c|}
\hline Study or Subgroup & $\begin{array}{l}\text { TA+GP } \\
\text { Events }\end{array}$ & $\begin{array}{l}\text { Is } \\
\text { Total }\end{array}$ & $\begin{array}{r}\mathrm{PCl} \\
\text { Events }\end{array}$ & Total & Weight & $\begin{array}{l}\text { Odds Ratio } \\
\text { M-H. Fixed, } 95 \% \mathrm{Cl}\end{array}$ & & $\begin{array}{r}\text { Odds } \\
\text { M-H. Fixe }\end{array}$ & $\begin{array}{l}\text { Ratio } \\
\text { ed. } 95 \% \mathrm{Cl}\end{array}$ & \\
\hline Choi et al.2009 & 5 & 10 & 71 & 113 & $16.1 \%$ & $0.59[0.16,2.16]$ & & - & & \\
\hline Guo YS et al.2011 & 52 & 63 & 38 & 61 & $18.8 \%$ & $2.86[1.25,6.57]$ & & & & \\
\hline Huang S 2013 & 23 & 31 & 51 & 96 & $17.9 \%$ & $2.54[1.03,6.23]$ & & & & \\
\hline Huang WG et al.2010 & 30 & 42 & 18 & 42 & $14.3 \%$ & $3.33[1.35,8.25]$ & & & & \\
\hline Wang XM et al.2012 & 25 & 32 & 14 & 32 & $8.5 \%$ & $4.59[1.54,13.67]$ & & & & \\
\hline Liu CP et al.2013 & 19 & 28 & 10 & 23 & $9.8 \%$ & $2.74[0.87,8.62]$ & & & & \\
\hline Zhou DH et al.2012 & 79 & 86 & 62 & 79 & $14.6 \%$ & $3.09[1.21,7.93]$ & & & & \\
\hline Total $(95 \% \mathrm{Cl})$ & & 292 & & 446 & $100.0 \%$ & $2.68[1.85,3.87]$ & & & & \\
\hline Total events & 233 & & 264 & & & & & & & \\
\hline \multicolumn{7}{|c|}{$\begin{array}{l}\text { Heterogeneity: } \mathrm{Chi}^{2}=6.50, \mathrm{df}=6(P=0.37) ;\left.\right|^{2}=8 \% \\
\text { Test for overall effect: } Z=5.24(P<0.00001)\end{array}$} & 0.01 & $\begin{array}{l}0.1 \\
\text { Favours [PCI] }\end{array}$ & 1 Favours [T & $\begin{array}{cc}10 & 100 \\
{[T A+I C} & \text { GPIs] }\end{array}$ \\
\hline
\end{tabular}

(b)

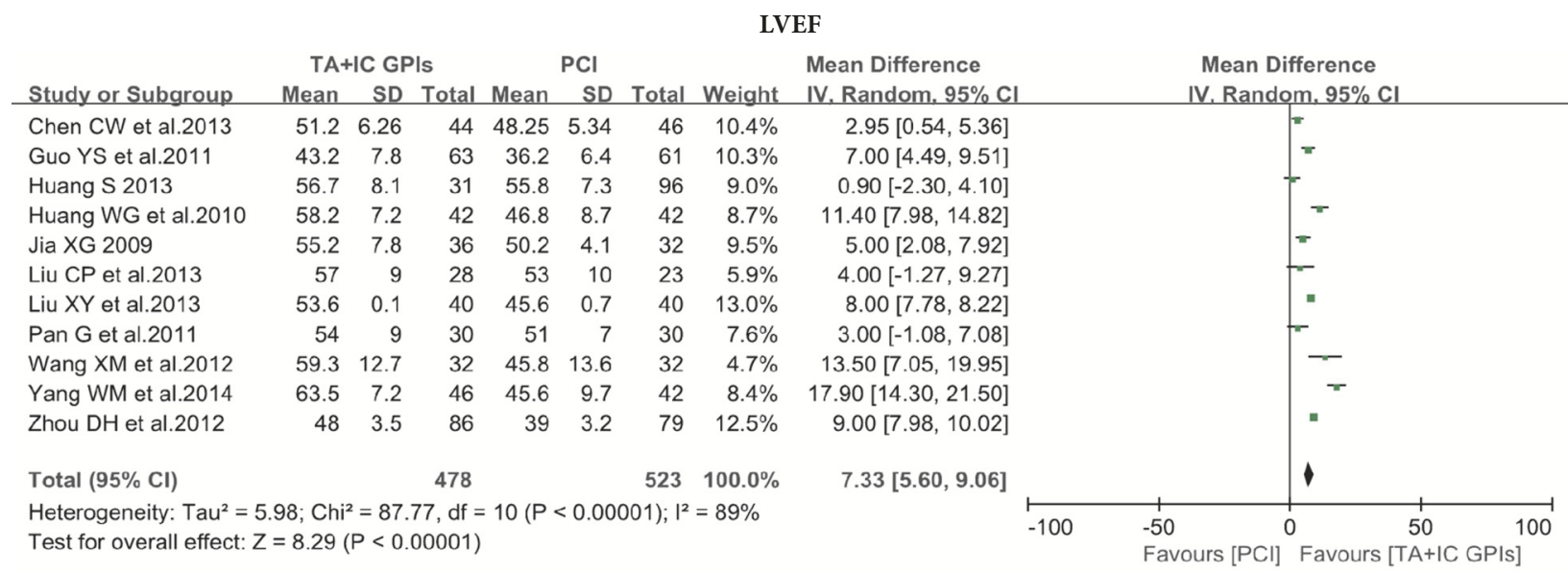

(c)

Figure 4: (a) Meta-analysis of postoperative TIMI grade 3 flow between thrombus aspiration plus intracoronary GPIs and PCI alone; (b) meta-analysis of postoperative complete ST resolution (STR) between thrombus aspiration plus intracoronary GPIs and PCI alone; (c) metaanalysis of LVEF before discharge or at the short-term follow-up between thrombus aspiration plus intracoronary GPIs and PCI alone. TIMI: the Thrombolysis in Myocardial Infarction trial; LVEF: left ventricular ejection fraction; TA: thrombus aspiration; IC: intracoronary; GPIs: glycoprotein IIb/IIIa inhibitors; PCI: percutaneous coronary interventions. 


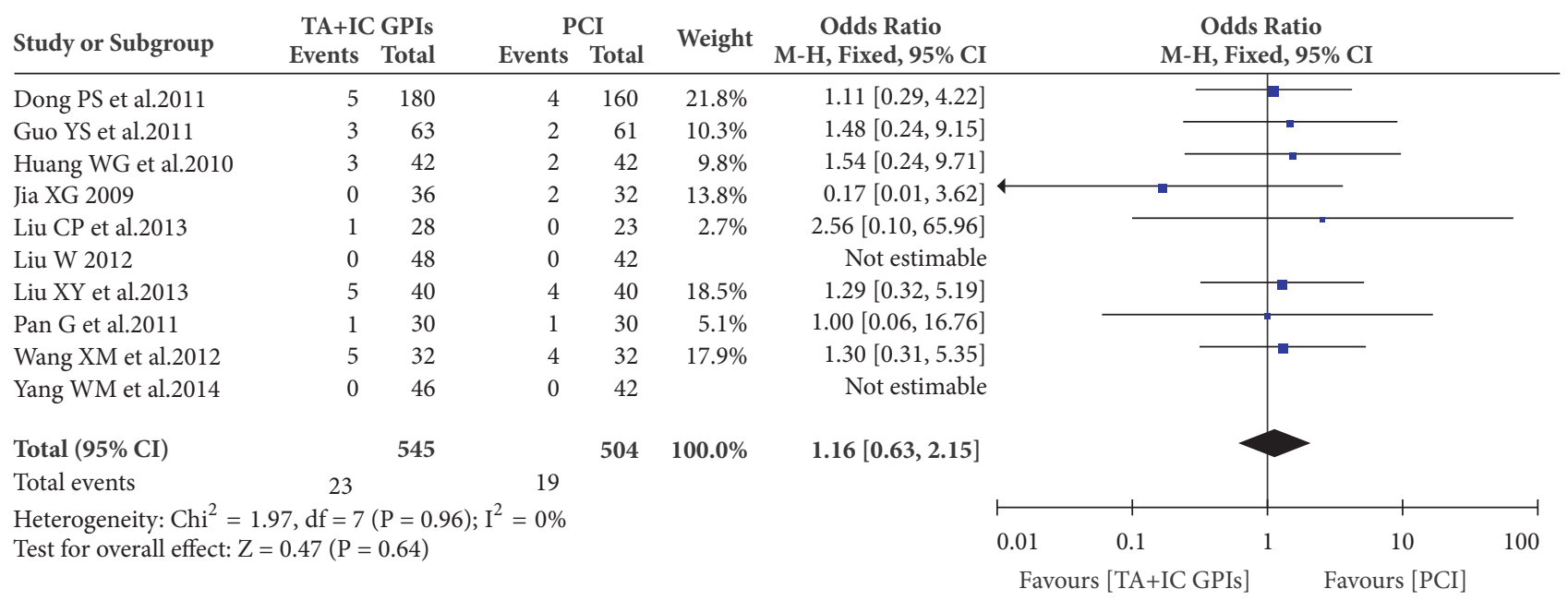

FIGURE 5: Meta-analysis of the major bleeding or minor bleeding events before discharge between thrombus aspiration plus intracoronary GPIs and PCI alone. TA: thrombus aspiration; IC: intracoronary; GPIs: glycoprotein IIb/IIIa inhibitors; PCI: percutaneous coronary interventions.

\section{Short-term MACE}

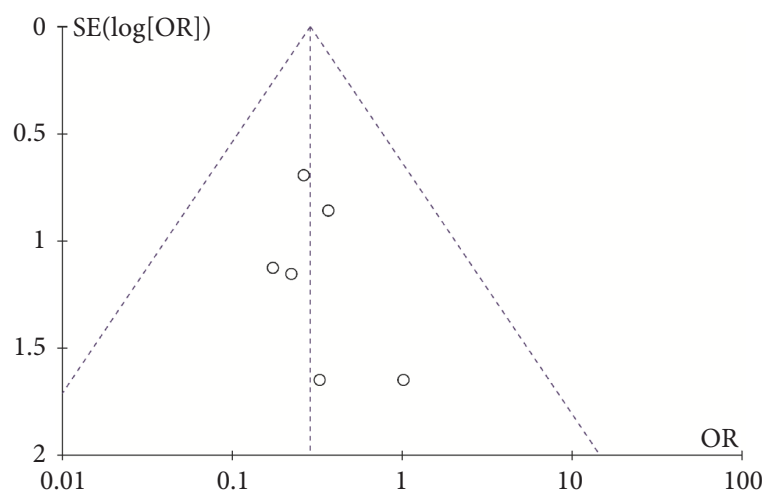

(a)

\section{Short-term death}

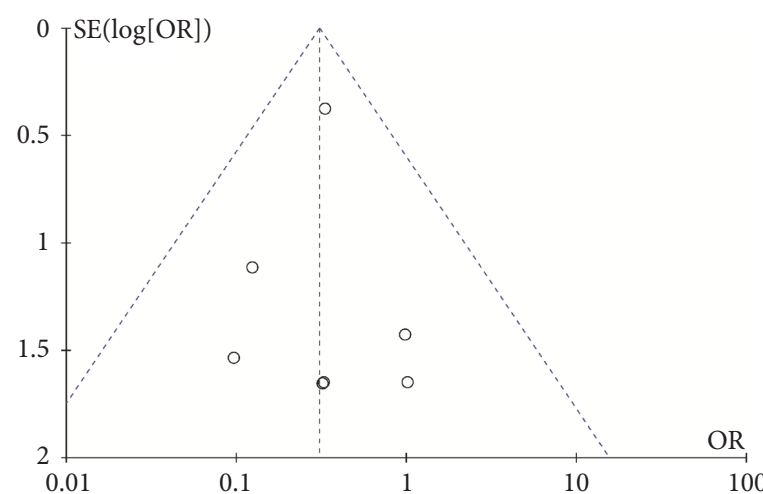

(b)

Short-term reinfarction

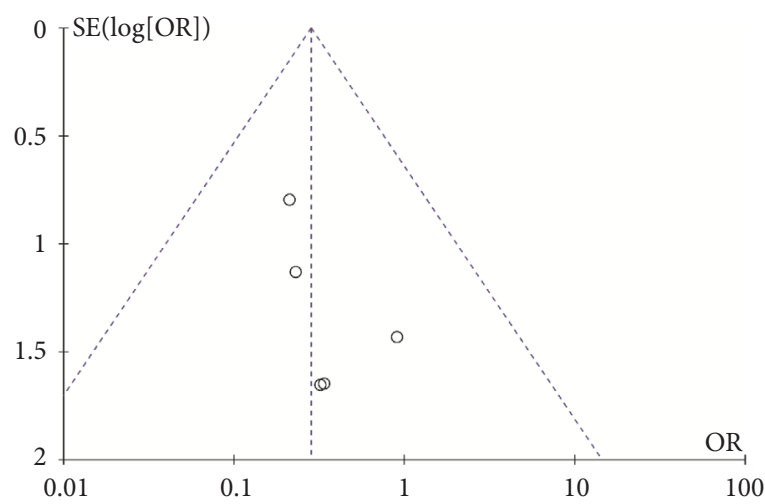

(c)

FIGURE 6: Funnel plot of publication bias for the short-term MACE (a), death (b), and reinfarction (c). MACE: major adverse cardiac events.

This might interpret why our results of the meta-analysis were different from other studies $[4,5]$. Notably, subgroup analysis showed that the type of aspiration catheter might influence the clinical outcomes and, in this aspect,
ZEEK catheter and EXPORT catheter, which present a stronger aspiration capacity for moderate to large thrombi, were superior to Driver C.E. catheter [28]. Differences in aspiration capacity between ZEEK, EXPORT, and Diver 
C.E. in this setting might influence the short-term outcome.

We found there was no significant difference between the number of bleeding events in the intracoronary GPIs group and those in the control group, despite GPIs' antiplatelet activity and the risk of bleeding. This might be due to the type of GPIs-tirofiban, which was mostly used in our metaanalysis (92.9\% used tirofiban). Tirofiban is a representative of small molecule glycoprotein IIb/IIIa inhibitor with reliable platelet inhibition and reversibility [29]. Tirofiban, the most applied glycoprotein IIb/IIIa inhibitor in East Asia now, was found in 13 included studies (Choi's study not mentioned due to the abstract in ANGIOPLASTY SUMMIT).

There were also some limitations in this meta-analysis. First, few studies were found in other countries of East Asia, and we could not perform analyses in other populations. Second, we calculated the $\mathrm{Nfs} 0.05$ to assess the publication bias and found the results of long-term MACE, death, and reinfarction were $-0.99,-0.99$, and -0.82 , which indicated publication bias and might influence the meta-analysis results. Third, $92.9 \%$ studies in our meta-analysis used tirofiban, and subgroup analysis of different type of GPIs was unable to be performed.

\section{Conclusions}

Our study demonstrates that intracoronary use of glycoprotein IIb/IIIa inhibitors may have a synergistic effect with thrombus aspiration on short-term mortality, reinfarction, and cardiac functional recovery. Future RCTs are needed to assess the impact of concomitant glycoprotein IIb/IIIa inhibitors with thrombus aspiration on the long-term outcomes of patients with STEMI.

\section{Data Availability}

The datasets generated and/or analyzed during the current study are available from the corresponding author on reasonable request.

\section{Conflicts of Interest}

The authors have no conflicts of interest to declare.

\section{Authors' Contributions}

Jia-hong $\mathrm{Wu}$ and Yu-guo Chen contributed to the study concept and design. Rui-jian Li and Pan-pan Hao drafted the manuscript. All authors revised the article and approved the final version to be published.

\section{Acknowledgments}

This study was supported by research grants from the Department of Health of Shandong Province (2013WS0216), the China Postdoctoral Science Foundation (2015M572054), and the Natural Science Foundation of Shandong Province (ZR2015HM039).

\section{References}

[1] G. W. Stone, "Angioplasty strategies in ST-segment-elevation myocardial infarction: Part I: Primary percutaneous coronary intervention," Circulation, vol. 118, no. 5, pp. 538-551, 2008.

[2] A. Durante and P. G. Camici, "Novel insights into an 'old' phenomenon: the no reflow," International Journal of Cardiology, vol. 187, pp. 273-280, 2015.

[3] P. J. Vlaar, T. Svilaas, I. C. van der Horst et al., "Cardiac death and reinfarction after 1 year in the Thrombus Aspiration during Percutaneous coronary intervention in Acute myocardial infarction Study (TAPAS): a 1-year follow-up study," The Lancet, vol. 371, no. 9628, pp. 1915-1920, 2008.

[4] B. Lagerqvist, O. Fröbert, G. K. Olivecrona et al., "Outcomes 1 year after thrombus aspiration for myocardial infarction," The New England Journal of Medicine, vol. 371, no. 12, pp. 1111-1120, 2014.

[5] S. S. Jolly, J. A. Cairns, S. Yusuf et al., "Randomized trial of primary PCI with or without routine manual thrombectomy," The New England Journal of Medicine, vol. 372, no. 15, pp. 13891398, 2015.

[6] G. W. Stone, A. Maehara, B. Witzenbichler et al., "Intracoronary abciximab and aspiration thrombectomy in patients with large anterior myocardial infarction: The INFUSE-AMI randomized trial," Journal of the American Medical Association, vol. 307, no. 17, pp. 1817-1826, 2012.

[7] C.-P. Liu, M.-S. Lin, Y.-W. Chiu et al., "Additive benefit of glycoprotein IIb/IIIa inhibition and adjunctive thrombus aspiration during primary coronary intervention: Results of the Initial Thrombosuction and Tirofiban Infusion (ITTI) trial," International Journal of Cardiology, vol. 156, no. 2, pp. 174-179, 2012.

[8] R. Li, P. Hao, Y. Chen, and Y. Zhang, "Association of cystatin C level and cardiovascular prognosis for patients with preexisting coronary heart disease: A meta-analysis," Chinese Science Bulletin, vol. 59, no. 5-6, pp. 539-545, 2014.

[9] J. Wang, J. Liu, X. Ni et al., "Adjuvant therapy of oral chinese herbal medicine for menopausal depression: A systematic review and meta-analysis," Evid Based Complement Alternat Med, 2018.

[10] C. W. Chen, Y. Cheng, R. L. Zheng, H. Ding, and Z. Y. Zhang, "Effect of Thrombus Aspiration With Intrainfarct-related Artery Tirofiban Injection on Cardiac Function and Noreflow in Patients With Acute ST-elevation Myocardial InfarctionCirculation Journal," Chinese Circulation Journal, vol. 28, no. 8, pp. 595-598, 2013.

[11] S. Huang, "Combination thrombus aspiration with tirofiban to patients with acute ST-elevation myocardial infarction," Anhui Medical and Pharmaceutical Journal, vol. 17, no. 7, pp. 1230-1231, 2013 (Chinese).

[12] W. G. Huang, T. G. Wu, Z. F. Jiang et al., "The efficiency and safety of the Zeek aspiration thrombectomy catheter and tirofiban in primary percutaneous coronary intervention of acute myocardial infarction," Chinese Journal of Cardiovascular Research, vol. 8, no. 7, pp. 481-483, 2010.

[13] X. G. Jia, "Zeek aspiration thrombectomy catheter and tirofiban in primary percutaneous coronary intervention," Practical Journal of Cardiac Cerebral Pneumal, vol. 17, no. 1, pp. 24-25, 2009.

[14] X. M. Wang, L. F. Du, B. H. Wu, L. Li, and H. L. Hou, "Thrombus Aspiration and intracoronary Tirofiban in primary percutaneous coronary intervention," World Health Digest Medical Periodical, vol. 9, no. 29, pp. 5-6, 2012. 
[15] P. S. Dong, S. Y. Xing, H. L. Wang, L. H. Lai, Z. J. Li, and C. Y. Li, "Application study of thrombus aspiration combined with Tirofiban in primary percutaneous coronary intervention for acute myocardial infarction," Clinical Cardiology Journal, vol. 27, no. 11, pp. 849-851, 2011.

[16] Y. S. Guo, F. Lin, XJ. Chen, and W. Chen, “Thrombus Aspiration and intracoronary Tirofiban in patients with acute myocardial infarction," Journal of China Traditional Chinese Medicine Information, vol. 3, no. 21, pp. 5-6, 2011.

[17] W. Liu, "The clinical analysis of thrombus aspiration catheter and tirofiban applying to emergency PCI technique," Chinese Journal of Cardiovascular Research, vol. 10, no. 7, pp. 505-507, 2012.

[18] G. Pan, X. P. Xi, X. J. Feng, S. C. Long, and Q. Luo, "Application of DIVER CE thrombus aspiration catheters combined with tirofiban in emergency percutaneous coronary intervention," Chinese Journal of Evidence-Based Cardiovascular Medicine, vol. 3, no. 3, pp. 186-188, 2011.

[19] W. M. Yang, S. B. Li, D. C. Xiang, W. S. Ou, and Y. B. Zhong, "The application of thrombus aspiration catheter combined with tirofiban in the treatment of percutaneous coronary intervention," China Modern Medicine, vol. 21, no. 8, pp. 41-43, 2014 (Chinese).

[20] D. H. Zhou, Y. Z. Jin, Q. Wang, F. C. Yao, and J. Liu, "Combination Thrombus Aspiration with Tirofiban to patients with Acute ST-elevation Myocardial Infarction performing percutaneous coronary intervention," Shandong Medical, vol. 52, no. 5, pp. 4445, 2012.

[21] X. Liu, P. Dong, S. Xing et al., "Clinical evaluation of thrombus aspiration combined with tirofiban in patients with acute myocardial infarction with elective percutaneous coronary intervention," Journal of International Medical Research, vol. 41, no. 5, pp. 1532-1540, 2013.

[22] Y. S. Choi, W. S. Chung, C. S. Park, H. J. Park, P. J. Kim, and K. B. Seung, "AS-8: Angiographic Improvement after Thrombus Aspiration Concomitant with Glycoprotein IIb/IIIa Inhibitor Therapy Does Not Affect Long-Term Mortality during Primary PCI," American Journal of Cardiology, vol. 103, no. 9, p. 4B, 2009.

[23] N. S. Bajaj, S. Ather, S. Gaba et al., "Glycoprotein IIb/IIIa inhibitors improve mortality after aspiration thrombectomy in patients with ST-segment elevation myocardial infarction," International Journal of Cardiology, vol. 187, no. 1, pp. 206-207, 2015.

[24] S. G. Ahn, S.-H. Lee, J. H. Lee et al., "Efficacy of combination treatment with intracoronary abciximab and aspiration thrombectomy on myocardial perfusion in patients with STsegment elevation myocardial infarction undergoing primary coronary stenting," Yonsei Medical Journal, vol. 55, no. 3, pp. 606-616, 2014.

[25] Y. J. Shimada, N. C. Nakra, J. T. Fox, and Y. Kanei, "Metaanalysis of prospective randomized controlled trials comparing intracoronary versus intravenous abciximab in patients with ST-elevation myocardial infarction undergoing primary percutaneous coronary intervention," American Journal of Cardiology, vol. 109, no. 5, pp. 624-628, 2012.

[26] A. Poli, R. Fetiveau, P. Vandoni et al., "Integrated analysis of myocardial blush and ST-segment elevation recovery after successful primary angioplasty: Real-time grading of microvascular reperfusion and prediction of early and late recovery of left ventricular function," Circulation, vol. 106, no. 3, pp. 313-318, 2002.
[27] F. Burzotta, M. De Vita, Y. L. Gu et al., "Clinical impact of thrombectomy in acute ST-elevation myocardial infarction: An individual patient-data pooled analysis of 11 trials," European Heart Journal, vol. 30, no. 18, pp. 2193-2203, 2009.

[28] H. J. Zhao, H. B. Yan, J. Wang et al., "Comparison of Diver CE and ZEEK manual aspiration catheters for thrombectomy in ST-segment elevation myocardial infarction," Chinese Medical Journal, vol. 122, no. 6, pp. 648-654, 2009.

[29] D. J. Schneider, "Anti-platelet therapy: Glycoprotein IIb-IIIa antagonists," British Journal of Clinical Pharmacology, vol. 72, no. 4, pp. 672-682, 2011. 


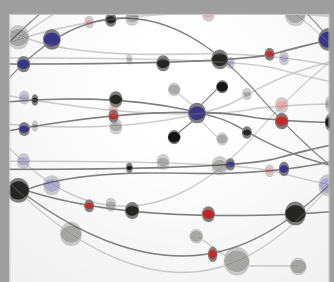

The Scientific World Journal
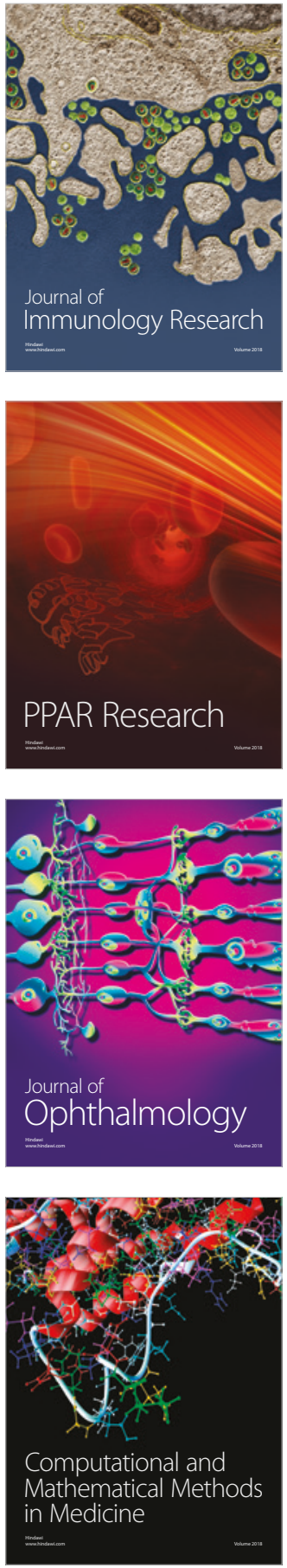

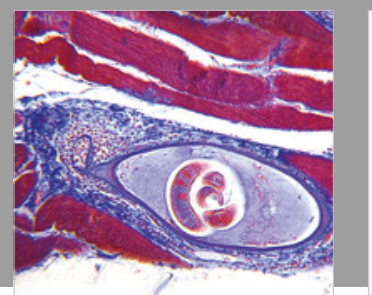

Gastroenterology Research and Practice

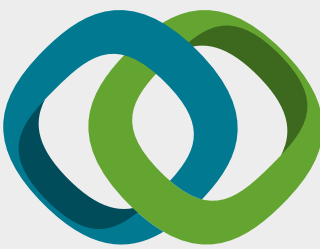

\section{Hindawi}

Submit your manuscripts at

www.hindawi.com
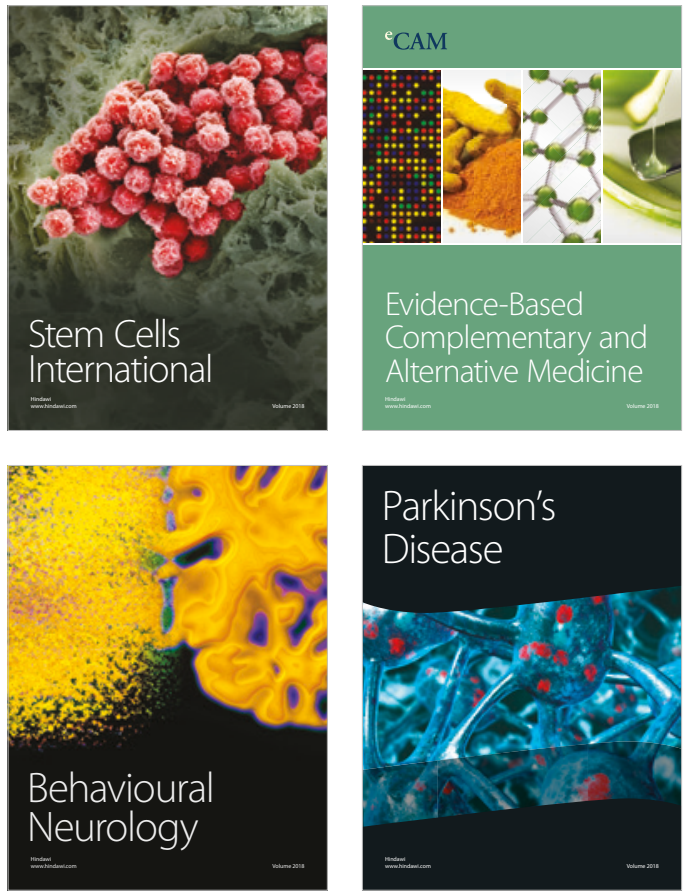

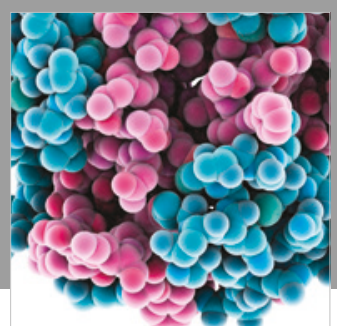

ournal of

Diabetes Research

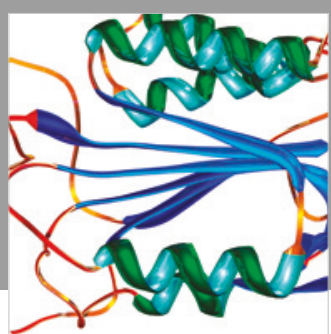

Disease Markers
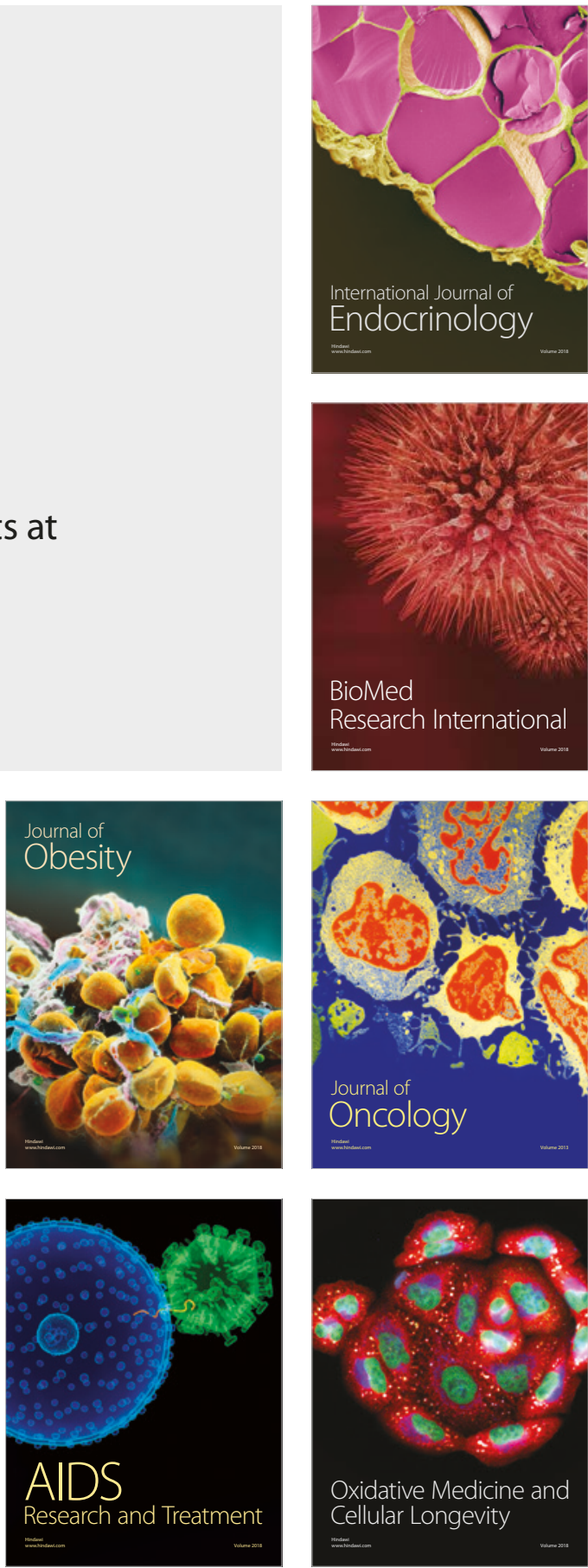